(2) Open Access Full Text Article

\title{
Comprehensive clinical and pathological analysis of aggressive renal epithelioid angiomyolipoma: report of three cases
}

This article was published in the following Dove Press journal:

OncoTargets and Therapy

27 May 2014

Number of times this article has been viewed

\author{
Jindan Luo' \\ Ben Liu' \\ Yanli Wang ${ }^{2}$ \\ Jun $\mathrm{Li}^{2}$ \\ Ping Wang' \\ Jun Chen' \\ Chaojun Wang' \\ 'Department of Urology, \\ ${ }^{2}$ Department of Pathology, The \\ First Affiliated Hospital of College \\ of Medicine, Zhejiang University, \\ Zhejiang, People's Republic of China
}

Correspondence: Chaojun Wang Department of Urology, The First Affiliated Hospital of College of Medicine, Zhejiang University, No 79 Qingchun Road, Hangzhou, Zhejiang 310003 ,

People's Republic of China

Email wangchaojun_zju@।63.com

\begin{abstract}
Renal angiomyolipoma (AML) is recognized as a benign hamartomatous lesion arising in the kidney with no obvious malignant potential. However, epithelioid AML (EAML), a rare variant of AML, is potentially malignant, with aggressive clinical features. It can occur in patients with or without tuberous sclerosis. Because EAML may mimic renal cell carcinoma in imaging studies, differentiation of this tumor from renal cell carcinoma preoperatively is difficult. At times, the lesions may extend into the renal vein and inferior vena cava or metastasize to other organs such as the lung and liver. To clarify the biological nature of EAML, three specific cases that we encountered in clinical practice are analyzed and reported in detail.
\end{abstract}

Keywords: kidney, malignant, inferior vena cava

\section{Introduction}

Renal angiomyolipoma (AML) is typically a solid lesion, composed of dysmorphic blood vessels, smooth muscle cells, and matured adipose tissue. AML is now deemed to belong to a family of neoplasms known as PEComas (perivascular epithelioid cell tumors). It has an incidence of about $0.3 \%-3.0 \%$, with a female preponderance. It occurs sporadically or in association with tuberous sclerosis. Epithelioid AML (EAML), first described in 1997 by Eble et al, ${ }^{1}$ is composed of epithelioid cells, polygonal cells, and varying degrees of nuclear atypia, with little or no fat cells. This rare subtype of AML is potentially malignant and may exhibit aggressive biological behavior, including recurrence, invasion into the inferior vena cava (IVC), and metastasis. Here, we report three cases of EAML with highly aggressive clinical features. The diagnosis and treatment of these cases are described in detail and discussed further.

\section{Case reports Case I}

A 27-year-old man with no stigmata of tuberous sclerosis complex (TSC) was referred with left flank dull pain and abdominal fullness for 5 days. Physical examination revealed a large mass in the left upper abdomen. Computed tomography (CT) scan demonstrated multiple masses in both kidneys. Of special interest was a large heterogeneous mass $(15.5 \mathrm{~cm} \times 13.0 \mathrm{~cm} \times 10.9 \mathrm{~cm})$ that occupied the upper middle portion of the left kidney (Figure 1). The average diameter was calculated by taking the average of three cases according to the longest diameter of the tumor (the third case taking the longest diameter of the primary tumor in the kidney). No metastatic disease was evident. All findings were diagnostic of bilateral renal AML, and a left nephrectomy was performed. 


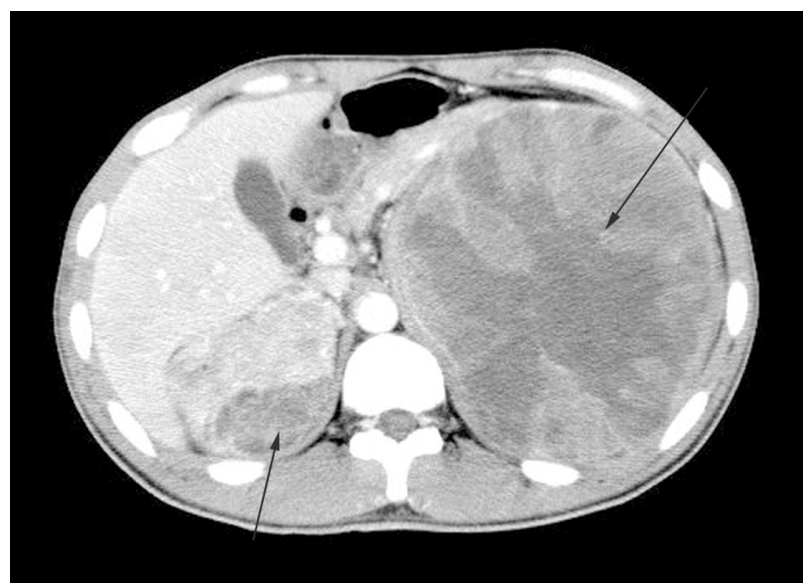

Figure I Abdominal computed tomography scan with intravenous contrast showing a large heterogeneous tumor with patchy areas of enhancement arising from the upper middle portion of the left kidney.

Notes: In addition, a non-homogeneous enhancement mass arises from the upper pole of the right kidney. The arrows indicate the lesion region.

Pathological analysis showed that the left renal tumor was composed predominantly (50\%-60\%) of epithelioid cells, with smooth muscle, blood vessels, and adipose tissue accounting for the remainder. The epithelioid cells had pleomorphic and hyperchromatic nuclei with densely eosinophilic cytoplasm (Figure 2A and B). Immunohistochemical studies showed the tumor cells to be positive for human melanosome-associated protein (HMB-45) (Figure 2C) and melanoma antigen recognized by T-cells 1 (MART1) (Figure 2D).

At 3 months postoperatively, the patient presented with fever and left flank pain. MRI (magnetic resonance imaging)
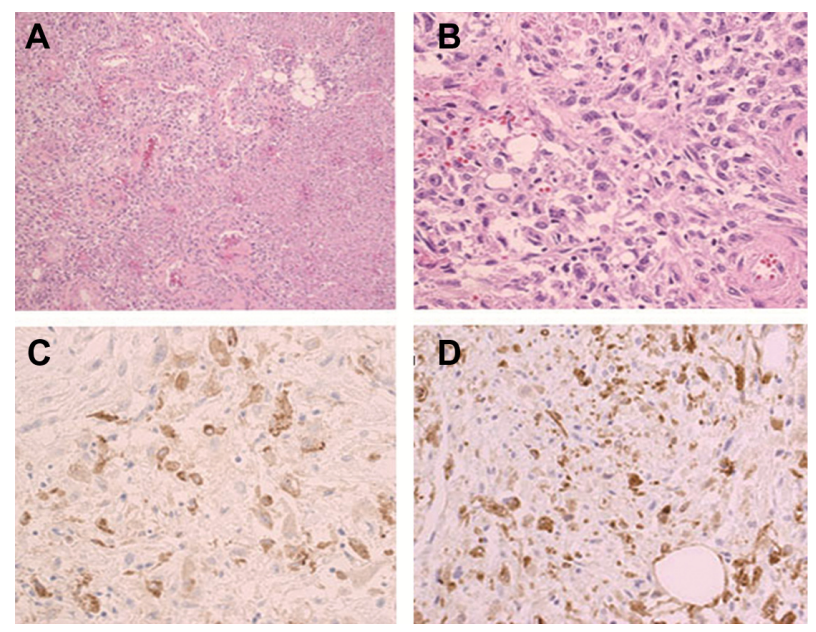

Figure 2 Histopathological findings of epithelioid angiomyolipoma.

Notes: (A) Microscopically, the tumor was composed of epithelioid cells, blood vessels, and adipose tissue (hematoxylin and eosin; magnification, $\times 100$ ). (B) Higher magnification shows that epithelioid cells exhibited pleomorphic and hyperchromatic nuclei with densely eosinophilic cytoplasm (hematoxylin and eosin; magnification, $\times 200$ ). (C) Tumor cells displayed strongly positive immunoreactivity for HMB-45 (magnification, $\times 200$ ). (D) Immunohistochemically, the tumor cells showed positive reactivity for MARTI (magnification, $\times 200$ ).

Abbreviation: MARTI, melanoma antigen recognized by T-cells I. revealed a large retroperitoneal mass in the left flank and multiple lesions in the liver. Chest X-ray examination showed left pleural effusion. The diagnosis was thought to be recurrent EAML with multiple hepatic metastases. We considered that the patient was not a surgical candidate because of the characteristics of the tumor and hepatic metastases. Thus, he was treated with conservative therapy. The patient's general state of health gradually deteriorated, and he died 4 months later.

\section{Case 2}

A 41-year-old man was referred to our institution complaining of having had left abdominal fullness for 2 months. The physical examination found a large mass in the left upper abdomen and no evidence of TSC. Routine laboratory investigations were within normal limits, except that urinalysis revealed $2+$ blood. CT angiography demonstrated a left renal mass $(17.0 \mathrm{~cm} \times 13.6 \mathrm{~cm} \times 9.2 \mathrm{~cm})$ with a tumor thrombus extending into the main renal vein and IVC (Figure 3A). A multifocal tumor ranging in diameter from 0.5 to $1.0 \mathrm{~cm}$ was also noted in the right kidney. All findings suggested the diagnosis of bilateral renal AML with the left renal vein and IVC invasion. No metastatic disease was evident. Thus, the patient underwent left radical nephrectomy and IVC thrombectomy.

Preoperative embolization of the left kidney was done, followed by subcostal transperitoneal incision and radical nephrectomy, with removal of the IVC thrombus. We fully mobilized the left kidney, and the tumor thrombus was identified in the main right renal vein and IVC. By mobilizing the liver off the IVC to the level of the main hepatic veins and using Satinsky clamps, vascular control of the IVC and right renal vein was achieved. The tumor thrombus was removed intact, and the IVC was repaired. Since the tumor thrombus did not adhere to the IVC wall, the cava wall resection was not required, and no enlarged lymph nodes were found.
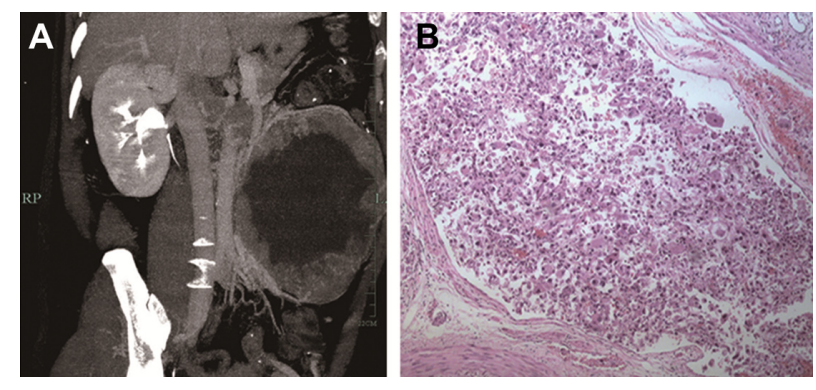

Figure 3 Radiological and histopathological findings of epithelioid angiomyolipoma. Notes: (A) A $17 \mathrm{~cm}$ left renal mass with a tumor thrombus extending into the main renal vein and inferior vena cava. (B) Tumor thrombus extending into the renal vein (hematoxylin and eosin; magnification, $\times 100$ ). 
Microscopic examination showed mature fatty tissue, a few vessels, and irregular bundles of the smooth muscle cells around the blood vessels in a radial fashion; most were epithelioid cells. Tumor thrombus was seen in the renal vein and IVC (Figure 3B). Immunohistochemistry confirmed the diagnosis of EAML, with focal positivity for HMB-45 and melan-A. At present, 21 months after the operation, the patient is asymptomatic and tumor-free.

\section{Case 3}

A 55-year-old woman was hospitalized with left flank pain for 3 months. Sonography revealed a solid mass $(7.5 \mathrm{~cm} \times$ $7.0 \mathrm{~cm} \times 6.7 \mathrm{~cm}$ ) in the left kidney, and radical nephrectomy was performed in May 1994. The diagnosis of renal cell carcinoma (RCC) was made without immunohistochemical examination. Seven years later, CT showed a mass of $8.0 \mathrm{~cm} \times$ $7.0 \mathrm{~cm} \times 6.5 \mathrm{~cm}$ in the lower pole, with another tumor of $1.5 \mathrm{~cm}$ in the upper pole of the right lung. Lobectomy of the right lung was then performed. The morphologic features of lung lesion (Figure 4) were consistent with the features of the renal lesion (Figure 5). Both renal and pulmonary tumors were reevaluated by immunohistochemical assays. The results showed that the tumor cells of both specimens were positive for vimentin and HMB-45, but they were negative for cytokeratin and S-100 protein. The corrected diagnosis of malignant renal EAML with pulmonary metastases was made. No chemotherapy or radiotherapy was administered postoperatively. The patient died as a result of respiratory failure due to multiple pulmonary metastases about 15 years after nephrectomy. Partial information of case 3 was reported in preliminary communications. ${ }^{7}$

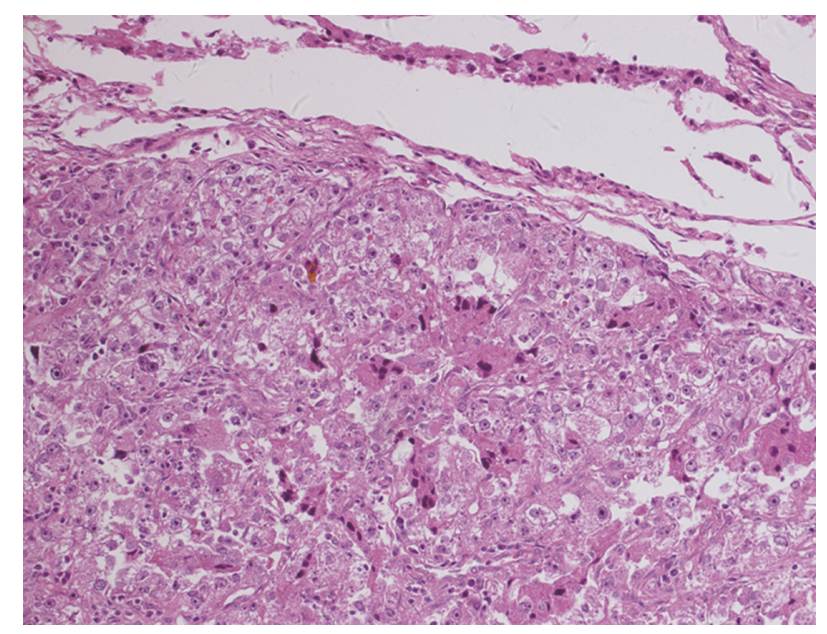

Figure 4 Histopathological findings of metastatic lesion in the lung.

Notes: The morpholgic features of lung lesion were consistent with the features of the renal lesion (hematoxylin and eosin; magnification, $\times 200$ ).

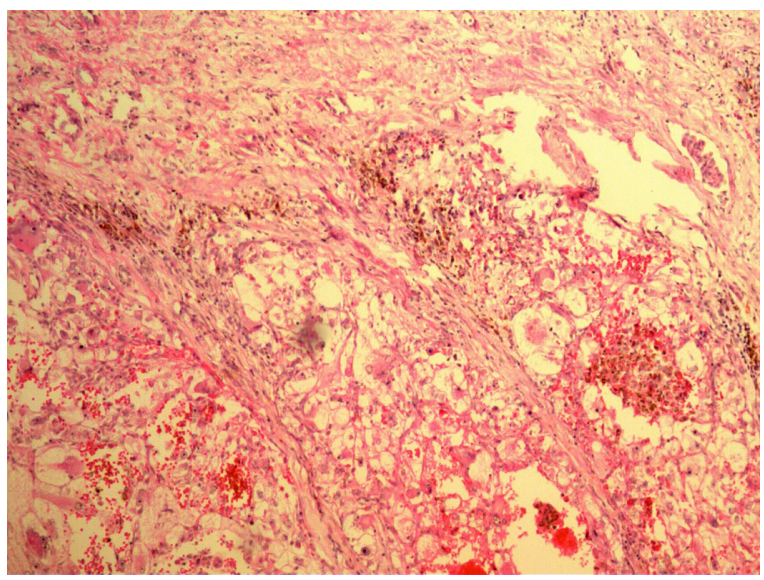

Figure 5 The original tumor in the kidney was composed of polygonal epithelioid cells with abundant eosinophilic cytoplasm, pleomorphic and hyperchromatic nuclei (hematoxylin and eosin; magnification, $\times 200$ ).

\section{Discussion}

EAML belongs to a family of mesenchymal tumors showing perivascular epithelioid cell differentiation. It is a subtype of AML in which epithelioid cells are mainly involved. Classical AMLs occur predominantly in females, with a ratio of 4:1 in both sporadic and TSC cases, while the ratio of occurrence of EAML in females versus males is 6.5:1.0. ${ }^{2}$ According to Aydin et al, ${ }^{2}$ patients with EAML are younger than classical AML patients (38.6 years versus 52.3 years), and the mean size of an EAML is also larger than that of a classical AML. EAML has been associated with aggressive clinical behavior, including extension into the vena cava and right atrium, and metastasis. Approximately $50 \%$ of reported patients develop metastatic lesions, and one-third of cases die as a result of the disease. ${ }^{3,4}$

Microscopically, EAML frequently presents with necrosis, hemorrhage, nuclear atypia, and mitotic activity and is thought to be a potentially malignant tumor. ${ }^{5-7}$ There are no histological criteria for malignant EAML, and different parameters have been proposed to predict malignancy in EAML. Large tumor size, necrosis, frequent mitoses, and atypical mitotic figures could be convincing criteria for malignancy. ${ }^{8}$ However, some investigators have suggested that only distant metastases can be accepted as a definite sign of malignancy. ${ }^{9}, 10$ According to scattered reports, the disease-specific survival for malignant EAML varies greatly (from 3 months to 9 years). ${ }^{3,4,9,10}$ The criteria for malignancy are still being developed. However, even without clinical and histopathological signs of malignancy, there are a few patients with EAML who exhibit highly aggressive biologic characteristics. EAML extending into the renal vein and IVC has been frequently reported. ${ }^{6,11,12}$ Yamamoto et a ${ }^{10}$ reported 
one patient with EAML who died 3 months after presentation due to widespread metastasis. However, that patient had multiple liver metastases when they made the original clinical diagnosis. In case one, there were no signs of metastases before surgery, yet the patient died 4 months after the surgery due to metastases. Therefore, we believe that this patient may had the most invasive EAML among reported cases.

There are no specific features of EAML in terms of clinical manifestations and imaging modalities. According to its high cellular content and lack of fat, it may appear as a hyperattenuating mass, with variable enhancement on CT. Although the imaging features of classical AML have been well described in radiology literature, the diagnosis of EAML is sometimes difficult because renal EAML can have a range of imaging appearances and can be indistinguishable from high-grade or sarcomatous RCC and AML with minimal fat. Park et al ${ }^{13}$ suggested that the diagnosis of EAML should be considered in patients diagnosed with conventional RCC with predominantly sarcomatoid features, especially when patients have clinical stigmata of TSC. Tsukada et a ${ }^{14}$ concluded that the radiological appearance of most EAML has a tendency to be hyperattenuating on unenhanced CT images, with low intensities on T2-weighted images on magnetic resonance imaging. They can be heterogeneously solid, homogeneously solid, or a multilocular cystic lesion with massive hemorrhage. EAML should also be included in the differential diagnosis of tumors with spontaneous perirenal hematoma. The average size of all eight EAMLs in Tsukada et al's study was $7.4 \mathrm{~cm}$. The larger size of EAML might be related to their malignant potential. In our three cases, the average diameter was $13.3 \mathrm{~cm}$. With all the criteria, there still exists a surprisingly high chance that EAML has been misinterpreted as a carcinoma. Pea et $\mathrm{al}^{15}$ reassessed the histology of five tumors previously diagnosed as RCC and found that only one of them was confirmed to be a true RCC on review. Three of them showed a phenotype compatible with EAML, and two of these patients subsequently died from metastatic disease. The third patient in our series is a typical example of first-time misdiagnosis. Therefore, the immunohistochemical studies are useful because EAML cells are usually positive for melanogenesis markers such as HMB-45 and melan-A, but negative for cytokeratins and RCC marker.

EAML is thought to be a unique variant of AML mainly because of its potential malignance. There is no definite pathologic feature that can predict the risk of its clinically aggressive behavior. Brimo et $\mathrm{al}^{16}$ developed a predictive model of four atypical features to predict the increased risk of malignance; while Nese et $\mathrm{al}^{4}$ proposed five features to predict the malignant behavior of EAML. Table 1 shows the comparison of Brimo et al's model and Nese et al's model to predict the malignancy of EAML. Two key features, renal vein involvement and necrosis, were shared in these two predictive models.

Definite diagnosis and tumor resection at an early stage could be important in the management of patients with EAML. The management of EAML depends on the size of the tumor, the radiological features, and the histological appearance. As the tumor mimics RCC on imaging studies, most patients with EAML are treated with surgical excision. Actually, surgical excision is the gold standard treatment for EAML due to its malignant potential. Partial nephrectomy should be reserved for tumors $\leq 4 \mathrm{~cm}$ in size. For tumors $>4 \mathrm{~cm}$, radical nephrectomy should be performed. However, in our first case, tumors relapsed dramatically shortly after the surgery, despite extensive excision, and the patient died 7 months after presentation due to widespread metastasis. Similar cases have been reported by other authors, ${ }^{10,13}$ showing the highly aggressive nature of malignant EAML. Therefore, a close follow-up is necessary once the diagnosis of EAML is confirmed.

Besides surgery, EAMLs are considered chemosensitive. EAML has been reported to respond to doxorubicin. ${ }^{3}$ In other reports, systemic treatments have included dacarbazine, ifosfamide, cyclophosphamide, and cisplastin. ${ }^{13}$ Furthermore, the mechanistic target of rapamycin (mTOR) pathway was recently found to be activated in EAML, and use of an mTOR inhibitor such as everolimus has been reported to result in dramatic tumor response for malignant EAML. ${ }^{17}$

In conclusion, EAML is capable of aggressive clinical behavior and should therefore be regarded clinically closer to malignant kidney tumors than to typical AMLs in terms

Table I Comparison of Brimo et al and Nese et al's models to predict the malignancy of epithelioid AML

\begin{tabular}{ll}
\hline Brimo et al's model & Nese et al's model \\
\hline$\geq 70 \%$ atypical epithelioid cells & TSC and/or concurrent AML \\
$\geq 2$ mitotic figures per $10 \mathrm{hpf}$ & Tumor size $(>7 \mathrm{~cm})$ \\
Atypical mitotic figures & With carcinoma-like growth \\
Necrosis & $\begin{array}{l}\text { Involvement of perinephric fat } \\
\text { tissue and/or renal vein }\end{array}$ \\
& Necrosis \\
The presence of three or all & Low-risk group (0-I of the \\
of the features was highly & above parameters); intermediate- \\
predictive of malignant behavior & risk group (2-3 of the above \\
& parameters); high-risk group \\
& (4-5 of the above parameters) \\
\hline
\end{tabular}

Note: Data taken from Brimo et $\mathrm{al}^{16}$ and Nese et $\mathrm{al}^{4}$.

Abbreviation: AML, angiomyolipoma; IVC, inferior vena cava, HMB-45, human melanosome-associated protein; MARTI, melanoma antigen recognized by T-cells I; hpf, high power field. 
of behavior and treatment. Histologically, it may resemble conventional RCC and can be distinguished by staining for HMB-45. Due to the rarity of these neoplasms, definite criteria of malignancy have not yet been established. As a treatment strategy for EAML, surgical resection is justified because of its malignant potential. Since some cases exhibit highly aggressive biologic characteristics, a close follow-up would be beneficial after surgery.

\section{Acknowledgments}

This study was supported by grants from the Scientific Research Fund of Zhejiang Provincial Education Department (Grant No Y201120149), the Scientific Research Fund of the Health Bureau of Zhejiang Province (Grant No 2013KYB099), and the Scientific Research Fund of the Science Technology Department of Zhejiang Province (Grant No 2012R10034).

\section{Disclosure}

The authors report no conflicts of interest in this work.

\section{References}

1. Eble JN, Amin MB, Young RH. Epithelioid angiomyolipoma of the kidney: a report of five cases with a prominent and diagnostically confusing epithelioid smooth muscle component. Am J Surg Pathol. 1997;21: 1123-1130.

2. Aydin H, Magi-Galluzzi C, Lane BR, et al. Renal angiomyolipoma: clinicopathologic study of 194 cases with emphasis on the epithelioid histology and tuberous sclerosis association. Am J Surg Pathol. 2009;33: 289-297.

3. Cibas ES, Goss GA, Kulke MH, Demetri GD, Fletcher CD. Malignant epithelioid angiomyolipoma ("sarcoma ex angiomyolipoma") of the kidney: a case report and review of the literature. Am J Surg Pathol. 2001;25:121-126.
4. Nese N, Martignoni G, Fletcher CD, et al. Pure epithelioid PEComas (so-called epithelioid angiomyolipoma) of the kidney: a clinicopathologic study of 41 cases: detailed assessment of morphology and risk stratification. Am J Surg Pathol. 2011;35:161-176.

5. Moudouni SM, Tligui M, Sibony M, et al. Malignant epithelioid renal angiomyolipoma involving the inferior vena cava in a patient with tuberous sclerosis. Urol Int. 2008;80:102-104; discussion 104.

6. Bakshi SS, Vishal K, Kalia V, Gill JS. Aggressive renal angiomyolipoma extending into the renal vein and inferior vena cava - an uncommon entity. Br J Radiol. 2011;84:e166-e168.

7. Li J, Zhu M, Wang YL. Malignant epithelioid angiomyolipoma of the kidney with pulmonary metastases and p 53 gene mutation. World $J$ Surg Oncol. 2012;10:213.

8. Sato K, Ueda Y, Tachibana H, et al. Malignant epithelioid angiomyolipoma of the kidney in a patient with tuberous sclerosis: an autopsy case report with p53 gene mutation analysis. Pathol Res Pract. 2008;204:771-777.

9. L'Hostis H, Deminiere C, Ferriere JM, Coindre JM. Renal angiomyolipoma: a clinicopathologic, immunohistochemical, and follow-up study of 46 cases. Am J Surg Pathol. 1999;23:1011-1020.

10. Yamamoto T, Ito K, Suzuki K, Yamanaka H, Ebihara K, Sasaki A. Rapidly progressive malignant epithelioid angiomyolipoma of the kidney. J Urol. 2002;168:190-191.

11. Bharwani N, Christmas TJ, Jameson C, Moat N, Sohaib SA. Epithelioid angiomyolipoma: imaging appearances. Br J Radiol. 2009;82: e249-e252.

12. Luo D, Gou J, Yang L, Xu Y, Dong Q. Epithelioid angiomyolipoma with involvement of inferior vena cava as a tumor thrombus: a case report. Kaohsiung J Med Sci. 2011;27:72-75.

13. Park HK, Zhang S, Wong MK, Kim HL. Clinical presentation of epithelioid angiomyolipoma. Int J Urol. 2007;14:21-25.

14. Tsukada J, Jinzaki M, Yao M, et al. Epithelioid angiomyolipoma of the kidney: radiological imaging. Int J Urol. 2013;20:1105-1111.

15. Pea M, Bonetti F, Martignoni G, et al. Apparent renal cell carcinomas in tuberous sclerosis are heterogeneous: the identification of malignant epithelioid angiomyolipoma. Am J Surg Pathol. 1998;22:180-187.

16. Brimo F, Robinson B, Guo C, Zhou M, Latour M, Epstein JI. Renal epithelioid angiomyolipoma with atypia: a series of 40 cases with emphasis on clinicopathologic prognostic indicators of malignancy. Am J Surg Pathol. 2010;34:715-722.

17. Shitara K, Yatabe Y, Mizota A, Sano T, Nimura Y, Muro K. Dramatic tumor response to everolimus for malignant epithelioid angiomyolipoma. Jpn J Clin Oncol. 2011;41:814-816.
OncoTargets and Therapy

\section{Publish your work in this journal}

OncoTargets and Therapy is an international, peer-reviewed, open access journal focusing on the pathological basis of all cancers, potential targets for therapy and treatment protocols employed to improve the management of cancer patients. The journal also focuses on the impact of management programs and new therapeutic agents and protocols on

\section{Dovepress}

patient perspectives such as quality of life, adherence and satisfaction. The manuscript management system is completely online and includes a very quick and fair peer-review system, which is all easy to use. Visit http://www.dovepress.com/testimonials.php to read real quotes from published authors. 